\title{
Rapid Progression of Autosomal Dominant Polycystic Kidney Disease: Urinary Biomarkers as Predictors
}

\author{
A. Lianne Messchendorp ${ }^{a} \quad$ Esther Meijer $^{\mathrm{a}} \quad$ Folkert W. Visser $^{\mathrm{a}}$ \\ Gerwin E. Engels $^{b}$ Peter Kappert ${ }^{c}$ Monique Losekoot ${ }^{d}$ Dorien J.M. Peters ${ }^{\mathrm{e}}$ \\ Ron T. Gansevoort ${ }^{\mathrm{a}}$ on behalf of the DIPAK-1 study investigators \\ ${ }^{a}$ Department of Nephrology, University Medical Center Groningen, University of Groningen, Groningen, The Netherlands; \\ ${ }^{\mathrm{b}}$ HaemoScan BV, Groningen, The Netherlands; ${ }^{\mathrm{c} C e n t e r ~ f o r ~ M e d i c a l ~ I m a g i n g, ~ U n i v e r s i t y ~ M e d i c a l ~ C e n t e r ~ G r o n i n g e n, ~}$ \\ University of Groningen, Groningen, The Netherlands; 'Department of Clinical Genetics, Leiden University Medical Center,

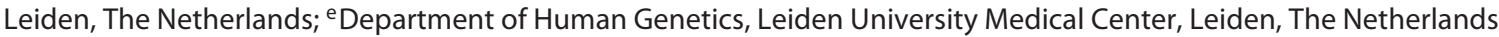

\section{Keywords}

Autosomal dominant polycystic kidney disease ·

Biomarkers · Chronic kidney disease progression · Beta-2

microglobulin - Monocyte chemotactic protein-1

\begin{abstract}
Background: Markers currently used to predict the likelihood of rapid disease progression in patients with autosomal dominant polycystic kidney disease (ADPKD) are expensive and time consuming to assess and often have limited sensitivity. New, easy-to-measure markers are therefore needed that alone or in combination with conventional risk markers can predict the rate of disease progression. In the present study, we investigated the ability of tubular damage and inflammation markers to predict kidney function decline. Methods: At baseline, albumin, immunoglobulin $\mathrm{G}$, kidney injury molecule $1, \beta 2$ microglobulin ( $\beta 2 M G$ ), heart-type fatty acid-binding protein, neutrophil gelatinase-associated lipocalin, and monocyte chemotactic protein-1 (MCP-1) were measured in 24-h urine samples of patients participating in a study investigating the therapeutic efficacy of lanreotide in ADPKD. Individual change in estimated glomerular filtration rate (eGFR)
\end{abstract}

during follow-up was calculated using mixed-model analysis taking into account 13 eGFRs (chronic kidney disease EPIdemiology) per patient. Logistic regression analysis was used to select urinary biomarkers that had the best association with rapidly progressive disease. The predictive value of these selected urinary biomarkers was compared to other risk scores using C-statistics. Results: Included were 302 patients of whom $53.3 \%$ were female, with an average age of $48 \pm 7$ years, eGFR of $52 \pm 12 \mathrm{~mL} / \mathrm{min} / 1.73 \mathrm{~m}^{2}$, and a height-adjusted total kidney volume (htTKV) of 1,082 $(736-1,669) \mathrm{mL} / \mathrm{m}$. At baseline, all urinary damage and inflammation markers were associated with baseline eGFR, also after adjustment for age, sex and baseline htTKV. For longitudinal analyses only patients randomized to standard care were considered $(n=152)$. A stepwise backward analysis revealed that $\beta 2 M G$ and MCP-1 showed the strongest association with rapidly progressive disease. A urinary biomarker score was created by summing the ranking of tertiles of $\beta 2 M G$ and MCP-1 excretion. The predictive value of this urinary biomarker score was higher com-

Principal investigators of the DIPAK-1 study are Joost P.H. Drenth, Johan W. de Fijter, Ron T. Gansevoort, Esther Meijer, Folkert W. Visser, Jack F.M. Wetzels, and Robert Zietse.

\begin{tabular}{ll}
\hline KARGER & The Author(s) \\
& Published by S. Karger AG, Basel Oparger \\
E-Mail karger@karger.com & This article is licensed under the Creative Commons Attribution- \\
www.karger.com/ajn & $\begin{array}{l}\text { NonCommercial-NoDerivatives 4.0 International License (CC BY- } \\
\text { NC-ND) (http://www.karger.com/Services/OpenAccessLicense). } \\
\text { Usage and distribution for commercial purposes as well as any dis- } \\
\text { tribution of modified material requires written permission. }\end{array}$
\end{tabular}

Prof. Dr. Ron T. Gansevoor

Department of Nephrology, University Medical Center Groningen

Expertise Center for Polycystic Kidney Diseases

PO 30.001, NL-9700 RB Groningen (The Netherlands)

E-Mail R.T.Gansevoort@umcg.nl 
pared to that of the Mayo htTKV classification (area under the curve [AUC] $0.73[0.64-0.82]$ vs. $0.61[0.51-0.71], p=0.04$ ) and comparable to that of the predicting renal outcomes in ADPKD score (AUC 0.73 [0.64-0.82] vs. 0.65 [0.55-0.75], $p=0.18$ ). In a second independent cohort with better kidney function, similar results were found for the urinary biomarker score. Conclusion: Measurement of urinary $\beta 2 \mathrm{MG}$ and MCP-1 excretion allows selection of ADPKD patients with rapidly progressive disease, with a predictive value comparable to or even higher than that of TKV or PKD mutation. Easy and inexpensive to measure urinary markers therefore hold promise to help predict prognosis in ADPKD.

The Author(s). Published by S. Karger AG, Basel

\section{Introduction}

Autosomal dominant polycystic kidney disease (ADPKD) is the most common inheritable kidney disease $[1,2]$. The disease is characterized by the formation and growth of cysts in both kidneys, which results in a decline in glomerular filtration rate (GFR). Ultimately, most subjects with ADPKD will reach end-stage kidney disease (ESKD). However, the age at which patients with ADPKD will reach ESKD shows large interindividual variability [3], even between family members that share the same mutation [4]. Predicting the rate of disease progression has become particularly important now with tolvaptan the first disease-modifying treatment for ADPKD has become available $[5,6]$. Especially patients with a high likelihood of rapid disease progression should be selected for this treatment because in such patients the benefit to risk ratio is expected to be optimal $[7,8]$.

Currently, several variables are available to predict the rate of disease progression in ADPKD. GFR indexed for age is a strong predictor but less sensitive in early stages of this disease, when GFR can remain in the normal range, while cysts are progressively formed [3]. Therefore, in early-stage disease, much attention has been focused on total kidney volume (TKV) as a predictor $[3,9,10]$. Disease progression is also influenced by the ADPKD genotype. Patients with a PKD1 mutation, especially truncating mutations, progress faster toward ESKD than patients with a PKD2 mutation $[4,11]$. However, assessment of TKV and genotype is laborious and expensive, and their associations with the rate of disease progression are limited at an individual patient level. Therefore, new risk markers need to be developed that alone, or in combination with conventional risk

(20)
DOI: $10.1159 / 000502999$ markers, can predict the rate of disease progression in ADPKD.

Previously, we showed that easy and inexpensive to measure urinary inflammation and damage markers have the potential to predict the rate of disease progression in ADPKD [12]. In this study, we aimed to confirm and extend these results by investigating in an independent cohort of ADPKD patients with standardized follow-up whether urinary tubular damage and inflammation markers can be used to select patients with rapidly progressive disease.

\section{Materials and Methods}

Setting and Subjects

For this study, we included subjects who participated in the DIPAK-1 study, which is an investigator-driven, prospective, randomized, controlled trial to test the efficacy and safety of lanreotide in later stage ADPKD [13]. For this trial, patients were included between 18 and 60 years of age, who had ADPKD based on the modified Ravine criteria [14], with an estimated GFR (eGFR) of $30-60 \mathrm{~mL} / \mathrm{min} / 1.73 \mathrm{~m}^{2}$. Main exclusion criteria of the DIPAK-1 study were bradycardia, a history of gallstones or pancreatitis, and diseases or medication use that could potentially affect kidney function (e.g., diabetes mellitus, or use of NSAIDs, lithium or tolvap$\tan$ ). ClinicalTrials.gov registration is NCT01616927. The study was approved by the institutional review boards of each study center. The study was performed in adherence to the Declaration of Helsinki, and all participants gave written informed consent.

\section{Clinical and Biochemical Measurements}

A detailed description of the study protocol has been published previously [13]. In short, patients were invited for a screening visit. When patients were eligible for study participation, a baseline visit took place. A day before the baseline visit, all patients collected $24-\mathrm{h}$ urine, of which samples were stored frozen at $-80^{\circ} \mathrm{C}$ until measurements took place. At the day of the baseline visit, blood was drawn for PKD mutation analyses and for creatinine measurement. Blood pressure was assessed at rest in a supine position with a semi-automatic, noninvasive sphygmomanometer (Dinamap) for $15 \mathrm{~min}$. Height and weight were measured and body mass index was calculated as weight in kilograms divided by height in square meters. Body surface area was calculated according to the DuBois formula [15]. PKD mutation analysis was performed with DNA isolation using PUREGENETM nucleic acid purification chemistry on the AUTOPURE LS 98 platform (Qiagen), followed by sequencing of amplified coding exons directly (exon 34-46), or on long-range PCR products (exon 1-33) [16]. After the baseline visit, patients were randomized to receive either standard care or lanreotide on top of standard care in a 1:1 ratio. Patients collected another 24-h urine after 12 weeks. Blood was drawn at screening, baseline, week 12 , and every 12 weeks thereafter until week 132 for the measurement of creatinine. Creatinine was measured with an enzymatic assay (isotope dilution mass spectrometry traceable; Modular, Roche Diagnostics). GFR was estimated (eGFR) for each time point with the 2009 chronic kidney disease EPIdemiology equation [17]. Magnetic
Messchendorp et al. 
resonance imaging (MRI) was performed at baseline and weeks 120 and 132 to assess TKV, using a standardized abdominal MRI protocol without the use of intravenous contrast. TKV was measured on T2-weighted coronal images using Analyze direct 11.0 (AnalyzeDirect, Inc., Overland Park, KS, USA) by classical volumetry (i.e., manual tracing) and adjusted for height TKV (htTKV).

The frozen 24-h urine samples from baseline and 12 weeks were thawed, and albumin was measured as general kidney damage marker; immunoglobulin $\mathrm{G}$ (IgG) as glomerular damage marker; $\beta 2 \mathrm{mi}$ croglobulin ( $\beta 2 \mathrm{MG})$ and kidney injury molecule 1 (KIM-1) as proximal tubular damage markers; heart-type fatty acid-binding protein (HFABP) as distal tubular damage marker; and neutrophil gelatinase-associated lipocalin (NGAL) and monocyte chemotactic protein-1 (MCP-1) as inflammation markers [18-26]. Urinary albumin was measured with a colorimetric assay using bromocresol green (BCG; Sigma Aldrich Co. LLC., St. Louis, MO, USA). Urinary IgG, HFABP (HyTest Ltd., Turku, Finland), $\beta 2 M G$ (Anogen-Yes Biotech Laboratories Ltd., Mississauga, Canada), KIM-1, NGAL, and MCP-1 (R\&D Systems Inc., Minneapolis, MN, USA) were measured by ELISA. Urine samples were diluted twice for KIM-1, $\beta 2 \mathrm{MG}$, and MCP-1, 5 times for HFABP, and 100 times for NGAL and IgG. Detection limit for albumin was $5.7 \mu \mathrm{g} / \mathrm{mL}$, for IgG $220 \mathrm{ng} / \mathrm{mL}$, for ß2MG $18 \mathrm{ng} / \mathrm{mL}$, for KIM-1 $0.087 \mathrm{ng} / \mathrm{mL}$, for HFABP $0.38 \mathrm{ng} / \mathrm{mL}$, for NGAL $22 \mathrm{pg} / \mathrm{mL}$, and for MCP-1 $0.04 \mathrm{ng} / \mathrm{mL}$. The intra- and interassay coefficients of variation were 3.8 and $6.3 \%$ for albumin, 8.4 and $16.4 \%$ for IgG, 6.3 and $8.5 \%$ for $\beta 2 \mathrm{MG}, 7.4$ and $14.5 \%$ for KIM$1,9.3$ and $17.6 \%$ for H-FABP, 5.2 and $19.6 \%$ for NGAL, and 8.3 and $12.7 \%$ for MCP-1, respectively. Biomarker excretions were calculated as urinary biomarker concentration $\times 24$-h urine volume.

\section{Statistical Analyses}

Normally distributed data are expressed as mean $\pm S D$, whereas nonnormally distributed data are expressed as median with interquartile range. Differences between patient groups were tested using a 2-sample $t$ test when normally distributed or a MannWhitney $U$ test when not normally distributed. A chi-square test was used in case of categorical data.

We tested the associations of baseline urinary biomarker excretions in all patients, and the intraindividual variation of urinary biomarker excretion at baseline and week 12 in patients randomized to standard care only with Pearson Correlation. With linear regression analysis, we investigated the association between baseline urinary biomarker excretion and eGFR and htTKV cross-sectionally in all patients. We tested associations crude and adjusted for age, sex and baseline htTKV in case of eGFR or baseline eGFR in case of htTKV.

For longitudinal analyses, we only considered patients who were randomized to standard care as lanreotide may influence disease progression. A mixed-model repeated measures analysis was used to calculate annual change in eGFR per patient. Patients were subsequently classified as having either rapidly or slowly progressive disease based on the average annual change in eGFR of the study population. With logistic regression analysis, we selected urinary biomarkers that had the best fit for rapidly progressive disease when variables were taken into account that are routinely available in clinical care (age, sex, and baseline eGFR). Associations of individual variables with rapidly progressive disease were tested with a Wald test, and the improvement of model fit was tested with the -2 log likelihood ratio test. We first investigated the improvement of model fit by adding a single urinary biomarker. Next, a stepwise backward analysis was performed to select the best performing urinary biomarkers. For this analysis, all urinary biomarkers were included plus the fixed variables age, sex, and baseline eGFR. In each step, the urinary biomarker with the weakest association was excluded until only urinary biomarkers remained with an $\alpha<0.1$.

With the urinary biomarkers that were selected by the stepwise backward analyses, we created a urinary biomarker score by summing the ranking of tertiles of these markers (with 1 the lowest and 3 the tertile with highest excretion). We first tested whether this score had an added predictive value (= area under the curve [AUC]) to a model with age, sex, and eGFR included using C-statistics. Next, we compared the predictive value of the urinary biomarker score to often used risk classifications for patients with ADPKD. We therefore classified patients according to the Mayo htTKV classification [10] and calculated the predicting renal outcomes in $\mathrm{AD}$ PKD (PROPKD) score [11]. We repeated these analyses, defining rapidly progressive disease based on the quartile with the most rapid annual change in eGFR of the study population.

Last, an independent cohort of ADPKD patients, with better preserved eGFR, was used to calculate a urinary biomarker score and we repeated our analysis using C-statistics. Details of this independent cohort and the description of the data collection have been published previously [12].

Analyses were performed with SPSS version 23.0 (SPSS Inc., Chicago, IL, USA) and STATA version 14 (Stata Corp., College Station, TX, USA). A two-sided $p<0.05$ was considered statistically significant.

\section{Results}

\section{Biomarker Excretions and Cross-Sectional \\ Associations}

For the cross-sectional analyses, we included 302 ADPKD patients of which $53.3 \%$ were female, who were $48.3 \pm 7.43$ years of age and had an eGFR of $51.6 \pm 11.6 \mathrm{~mL} /$ min/1.73 $\mathrm{m}^{2}$ (Table 1). Baseline urinary biomarkers excretions are displayed in Table 2. Correlations amongst the urinary biomarkers were high (online suppl. Table S1; for all online suppl. material, see www.karger.com/ doi/10.1159/000502999), and there was low intra-individual variation over time in all urinary biomarker excretions (online suppl. Fig. S1). All urinary damage and inflammation markers were associated with baseline eGFR, also after adjustment for age, sex, and baseline htTKV (online suppl. Table S2, model 2), and all markers, except $\beta 2 \mathrm{MG}$ and HFABP, were associated with baseline htTKV after adjustment for age, sex, and eGFR (online suppl. Table S3, model 2).

\section{Biomarker Excretions and Longitudinal Associations}

For the longitudinal analyses, we only included patients who received standard care at follow-up $(n=152)$. The average follow-up time was $2.43 \pm 0.41$ years in which 
Table 1. Baseline characteristics of ADPKD patients $(n=302)$

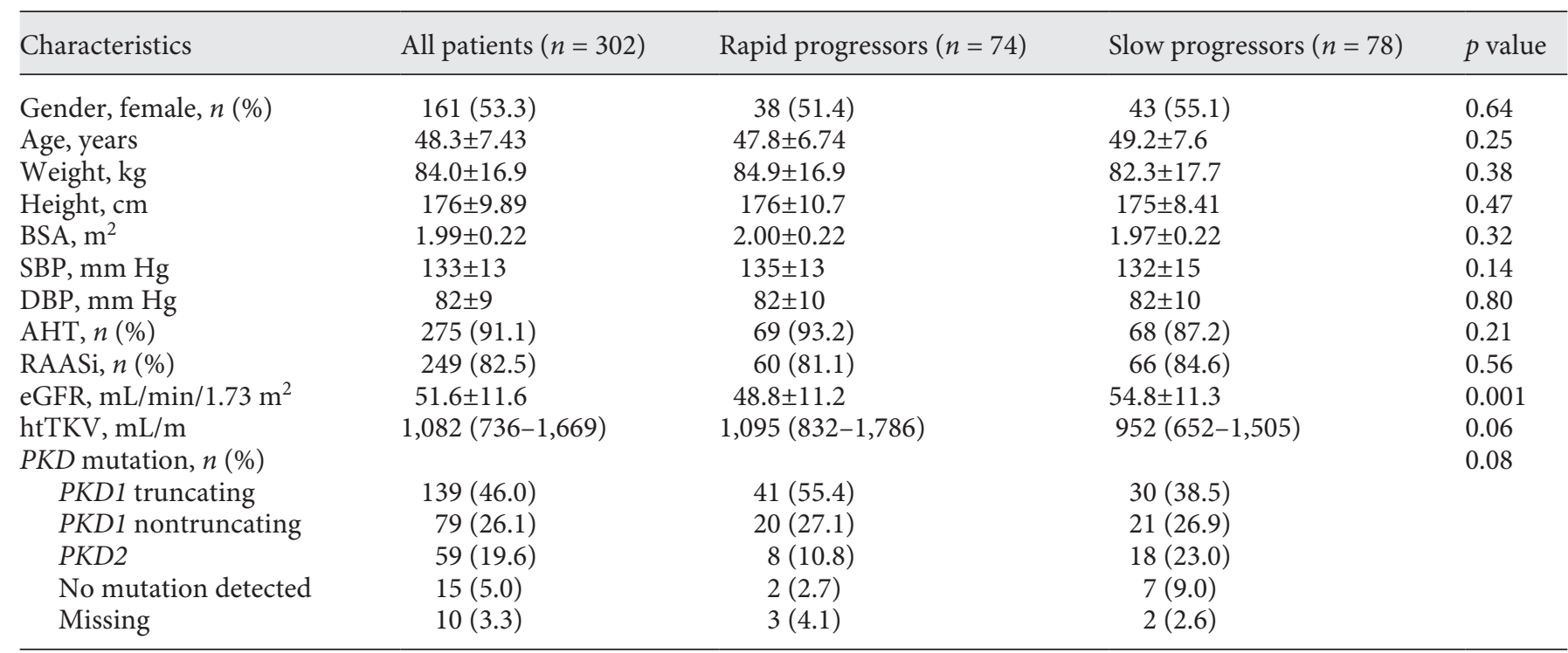

Variables are presented as mean \pm SD or as median (IQR) in case of nonnormal distribution.

$p$ values for fast versus slow progressors are calculated using independent-sample $t$ test in case of normal distribution, Mann-Whitney $U$ in case of nonnormal distribution, and chi-square in case of categorical data. Rapid and slow progressors were defined as patients with an annual change in eGFR less than or equal to -3.5 or greater than $-3.5 \mathrm{~mL} / \mathrm{min} / 1.73 \mathrm{~m}^{2}$, respectively.

ADPKD, autosomal dominant polycystic kidney disease; BSA, body surface area; SBP, systolic blood pressure; DBP, diastolic blood pressure; AHT, anti-hypertensive therapy; RAASi, RAAS inhibitors; eGFR, estimated glomerular filtration rate; htTKV, height-adjusted total kidney volume; PKD, polycystic kidney disease.

Table 2. Baseline urinary biomarker excretion $(n=302)$

\begin{tabular}{|c|c|c|c|c|}
\hline Urinary biomarker & All patients $(n=302)$ & Rapid progressors $(n=74)$ & Slow progressors $(n=78)$ & $p$ value \\
\hline \multicolumn{5}{|l|}{ General, mg/24 h } \\
\hline Albumin & $184.3(144.4-240.3)$ & $196.3(154.4-248.1)$ & $174.8(132.7-211.7)$ & 0.02 \\
\hline \multicolumn{5}{|l|}{ Glomerular, mg/24 h } \\
\hline $\operatorname{IgG}$ & $6.03(3.59-10.82)$ & $7.18(4.51-11.74)$ & $5.04(3.38-9.52)$ & 0.02 \\
\hline \multicolumn{5}{|c|}{ Proximal tubular, $\mu \mathrm{g} / 24 \mathrm{~h}$} \\
\hline$\beta 2 \mathrm{MG}$ & $191.2(120.9-258.4)$ & $247.0(168.8-307.8)$ & $183.1(87.7-239.0)$ & $<0.001$ \\
\hline \multicolumn{5}{|c|}{ Distal tubular, $\mu \mathrm{g} / 24 \mathrm{~h}$} \\
\hline HFABP & $17.6(12.2-25.4)$ & $21.9(13.4-31.6)$ & $17.0(12.4-22.8)$ & 0.005 \\
\hline \multicolumn{5}{|l|}{ Inflammatory } \\
\hline NGAL, $\mu \mathrm{g} / 24 \mathrm{~h}$ & $39.4(24.5-63.8)$ & $50.6(29.9-76.7)$ & $34.8(23.4-55.1)$ & 0.007 \\
\hline MCP-1, ng/24 h & $517.9(317.7-809.4)$ & $612.5(421.0-944.9)$ & $374.8(240.1-684.2)$ & 0.001 \\
\hline
\end{tabular}

Variables are presented as median (IQR) and $p$ values were calculated for rapid versus slow progressors with a Mann-Whitney U test. Rapid and slow progressors were defined as patients with an annual change in eGFR less than or equal to -3.5 or greater than $-3.5 \mathrm{~mL} /$ $\min / 1.73 \mathrm{~m}^{2}$, respectively.

IgG, immunoglobulin G; $\beta 2 \mathrm{MG}, \beta 2$ microglobulin; KIM-1, kidney injury molecule 1 ; HFABP, heart-type fatty acid-binding protein; NGAL, neutrophil gelatinase-associated lipocalin; MCP-1, monocyte chemotactic protein 1. 
Table 3. Model predicting rapidly versus slowly progressive disease (annual change in eGFR less than or equal to or greater than $-3.5 \mathrm{~mL} /$ $\left.\min / 1.73 \mathrm{~m}^{2} ; n=152\right)$

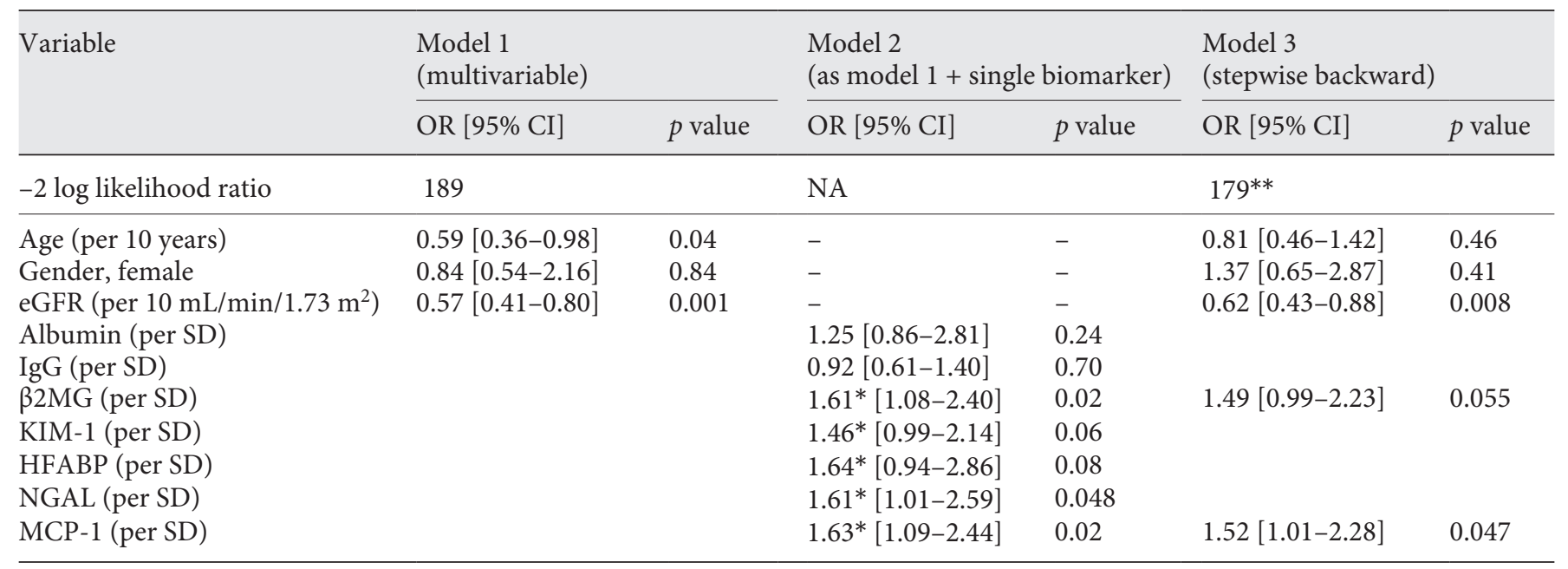

* $-2 \log$ likelihood ratio $p<0.05$ compared to model 1 .

** $-2 \log$ likelihood ratio $p=0.007$ compared to model 1 and $p=0.04$ and $p=0.049$ compared to model 2 with $\beta 2 \mathrm{MG}$ or MCP-1, respectively.

ORs and $p$ values were calculated using logistic regression analysis. Dependent variable is rapid versus slow disease progression (annual change in eGFR less than or equal to -3.5 versus greater than $-3.5 \mathrm{~mL} / \mathrm{min} / 1.73 \mathrm{~m}^{2}$ ).

Model 1: Age, female sex, and eGFR.

Model 2: Age, female sex, eGFR, and one of the urinary biomarkers.

Model 3: Age, female sex, eGFR, urinary $\beta 2 \mathrm{MG}$, and MCP-1 excretion.

eGFR, estimated glomerular filtration rate; IgG, immunoglobulin G; $\beta 2 \mathrm{MG}, \beta 2$ microglobulin; KIM-1, kidney injury molecule 1; HFABP, heart-type fatty acid-binding protein; NGAL, neutrophil gelatinase-associated lipocalin; MCP-1, monocyte chemotactic protein 1.

patients had an annual change in eGFR of $-3.52 \pm 2.01 \mathrm{~mL} /$ $\mathrm{min} / 1.73 \mathrm{~m}^{2}$ per year and an annual change in htTKV of $6.06 \pm 2.20 \%$ per year.

Online supplementary Table 44 shows results of a linear regression analysis with annual change in eGFR as dependent variable and the urinary biomarkers as independent variable. Crude, all markers were associated with annual change in eGFR. After adjustment for age, sex, baseline eGFR, htTKV, and PKD mutation, only $\beta 2 \mathrm{MG}$, KIM-1, HFABP, and MCP-1 remained significantly associated (online suppl. Table S4, model 3). None of the biomarkers was associated with annual change in htTKV, neither crude nor after adjustment for covariates (online suppl. Table S5).

\section{Biomarker Excretions and Prediction of Rapidly \\ Progressive Disease}

To select patients for treatment in clinical practice, it is important to distinguish patients with rapidly from slowly progressive disease. Patients were divided in either having rapidly $(n=74)$ or slowly progressive disease $(n=$ 78 ) based on the mean annual change in eGFR of the pop- ulation (less than or equal to -3.5 or greater than $-3.5 \mathrm{~mL}$ / $\mathrm{min} / 1.73 \mathrm{~m}^{2}$ per year). Other large-scale clinical trials reported a similar value for mean annual kidney function decline in patients with ADPKD $[5,6,27,28]$. Baseline characteristics were comparable between rapid and slow progressors except for eGFR and TKV (Table 1). All urinary biomarker excretions were higher in fast compared to slow progressors (Table 2).

Since in routine clinical practice information on age, sex, and eGFR is always available, we investigated which of the markers had the strongest association with rapidly progressive disease when added to these variables (Table $3)$. When adding $32 \mathrm{MG}$, KIM-1, HFABP, NGAL, or MCP-1 (model 2), the fit of the model improved significantly. When performing a stepwise backward analysis testing all urinary biomarkers, with age, sex, and eGFR as fixed variables, only $\beta 2 \mathrm{MG}$ and MCP-1 remained associated (model 3 ) and improved the model fit compared to model 1 and 2 . The fit of the model improved only further when $P K D$ mutation was added $(p=0.045)$ but not when baseline htTKV was added $(p=0.84)$. 

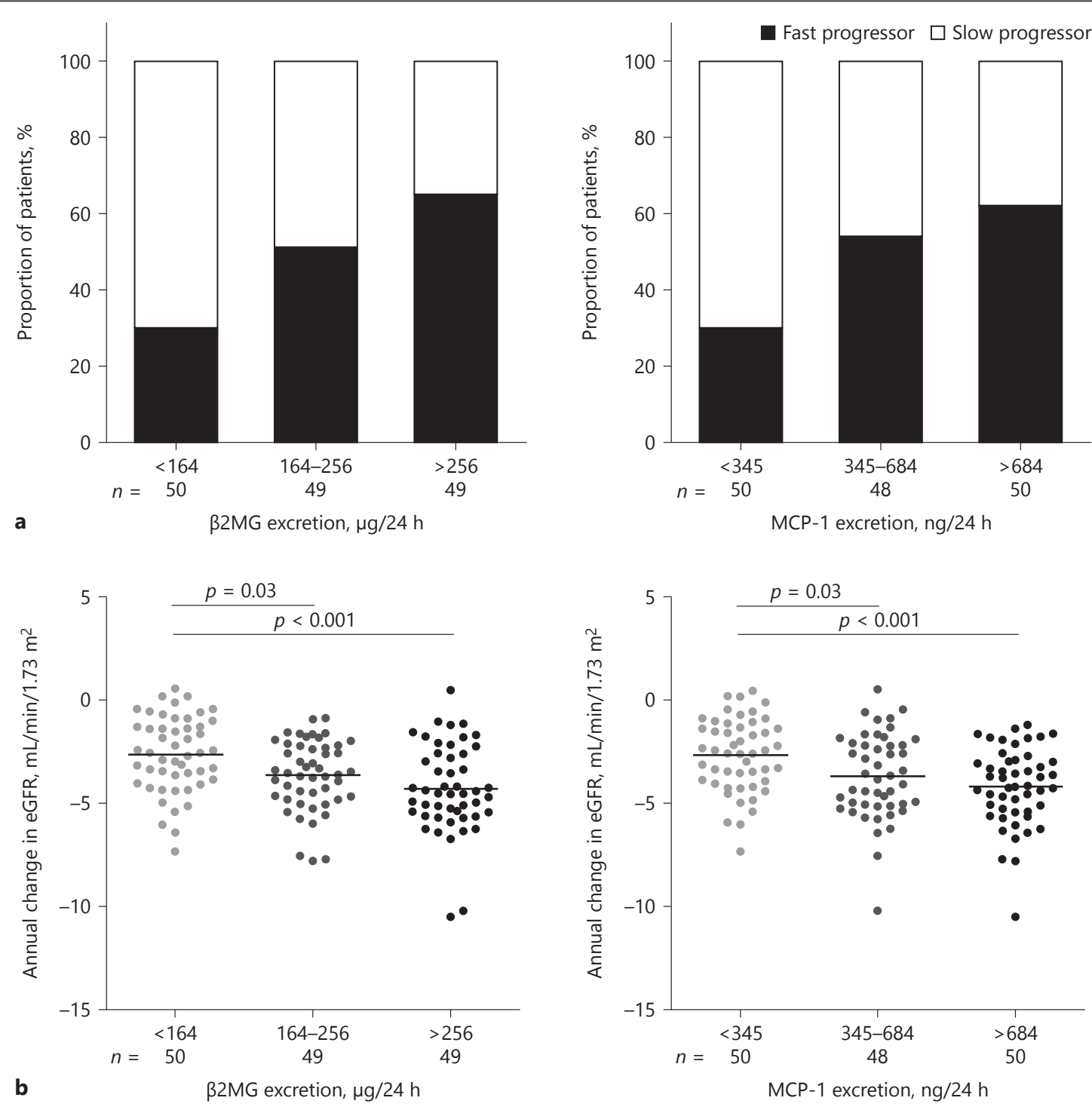

Fig. 1. Proportion of patients with rapidly progressive disease (a) and annual change in eGFR (b) according to tertiles of biomarker excretion. Rapid and slow progressors were defined as patients with an annual change in eGFR less than or equal to -3.5 or greater than $-3.5 \mathrm{~mL} / \mathrm{min} / 1.73 \mathrm{~m}^{2}$, respectively. Differences in propor-

tion across tertiles of $\beta 2 \mathrm{MG}$ excretion $p=0.002$ and across tertiles of MCP-1 excretion $p=0.004$. $\beta 2 \mathrm{MG}, \beta 2$ microglobulin; MCP-1, monocyte chemotactic protein-1; eGFR, estimated glomerular filtration rate.

Predictive Value of an Urinary Biomarker Score

Figure 1 displays the proportion of patients with fast or slow progressing disease and annual change in eGFR according to tertiles of $\beta 2 \mathrm{MG}$ excretion or MCP-1 excretion. Patients in the lowest tertile of $\beta 2 \mathrm{MG}$ or MCP-1 excretion had a significantly lower rate of eGFR decline compared to patients in the 2 higher tertiles. By summing the ranking of tertiles of these markers (with 1 the lowest and 3 the highest excretion), a urinary biomarker score was created with values ranging from 2 to 6 . With a lower score, a slower decline in eGFR was observed (Fig. 2). Patients with the lowest score of 2 had a significantly slower rate of eGFR decline compared to patients with a score of 5 or 6.

The urinary biomarker score improved the predictive value of a model with age, sex, and eGFR included (AUC 0.67 [0.60-0.75] vs. 0.73 [0.65-0.81], $p=0.049$; Fig. 3a). Subsequently, we compared the predictive value of the 


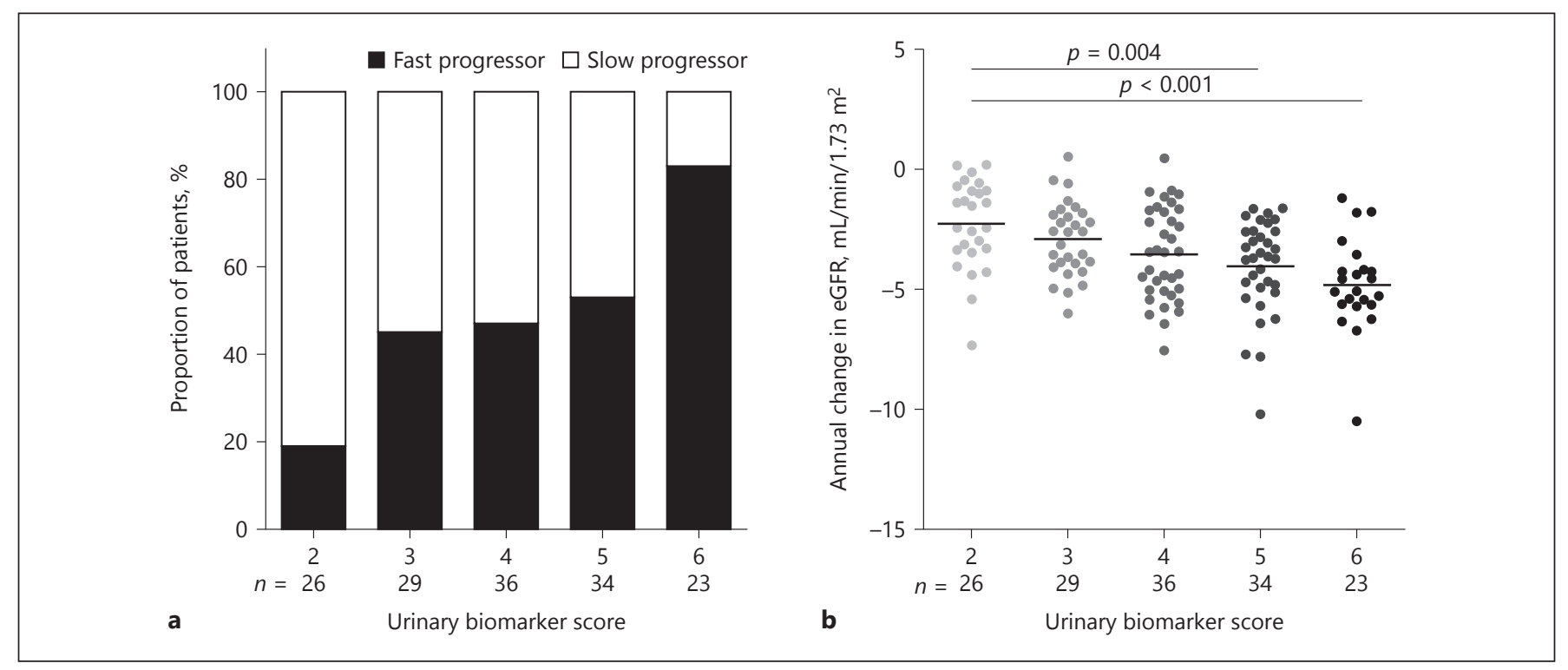

Fig. 2. Proportion of patients with rapidly progressive disease (a) and annual change in eGFR (b) according to a urinary biomarker score, calculated by combining tertiles of $\beta 2 \mathrm{MG}$ excretion and MCP-1 excretion. Rapid and slow progressors were defined as pa- tients with an annual change in eGFR less than or equal to -3.5 or greater than $-3.5 \mathrm{~mL} / \mathrm{min} / 1.73 \mathrm{~m}^{2}$, respectively. Differences in proportion across urinary biomarker score $p<0.001$. eGFR, estimated glomerular filtration rate.

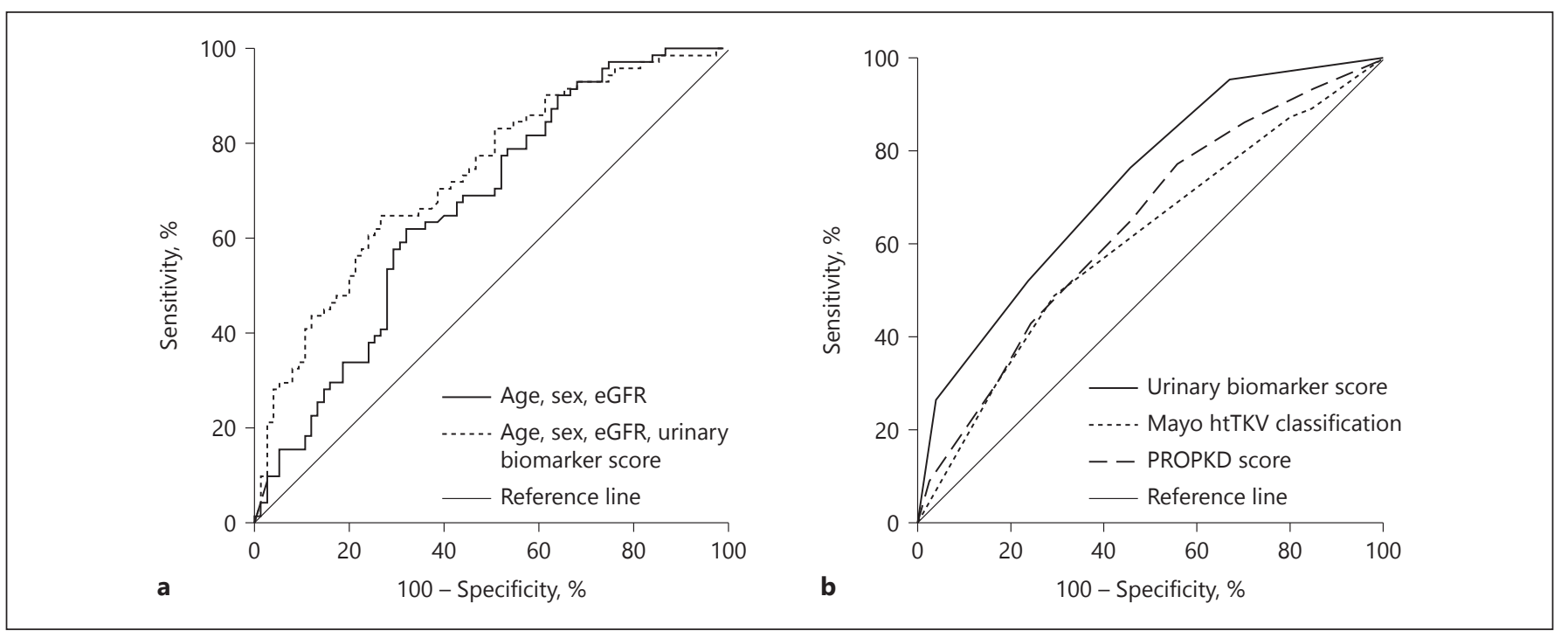

Fig. 3. Added predictive value of urinary biomarker score on top of age, sex, and eGFR (a; $n=146$; AUC 0.67 [0.58-0.75] vs. 0.73 [0.65-0.81], $p=0.049$ ) and predictive value of urinary biomarker score compared to Mayo htTKV classification or PROPKD score

urinary biomarker score with other, often used risk classifications for patients with ADPKD. We therefore classified patients according to their Mayo htTKV class and calculated the PROPKD score. We had htTKV available for 150 patients from which $5(3.3 \%)$ were classified as (b; $n=115$; AUC 0.73 [0.64-0.82] vs. 0.61 [0.51-0.71], $p=0.04$ or 0.65 [0.55-0.75], $p=0.18$ respectively). eGFR, estimated glomerular filtration rate; htTKV, height-adjusted total kidney volume; PROPKD, predicting renal outcomes in ADPKD. 
tients with typical ADPKD. The PROPKD score was available for 123 patients and $33.3 \%$ of these patients were classified as having a low risk (PROPKD score $\leq 3$ ), 44.7\% an intermediate risk (PROPKD score 4-6), and 22.0\% a high risk (PROPKD score 7-9). Online supplementary Figure S3 shows the proportion of patients with rapidly progressive disease according to this score. In our patients, the predictive value of the urinary biomarker score was higher than that of the Mayo htTKV classification (AUC $0.73[0.64-0.82]$ vs. $0.61[0.51-0.71], p=0.04$ ). The predictive value of the urinary biomarker score was also higher compared to that of the PROPKD score, but this did not reach statistical significance (AUC 0.73 [0.640.82 ] vs. 0.65 [0.55-0.75], $p=0.18$; Fig. $3 b$ ). Of note, the Mayo htTKV classification or the PROPKD score did not improve the prediction of rapidly progressive disease on top of age, sex, eGFR, and the urinary biomarker score (AUC $0.75[0.66-0.84]$ vs. $0.80[0.72-0.89], p=0.07$ and vs. $0.80[0.72-0.89], p=0.12$ ).

We repeated these analyses, defining rapid disease progression as the subjects belonging to the quartile with the most rapid annual change in eGFR (annual change in eGFR less than or equal to $-4.9 \mathrm{~mL} / \mathrm{min} / 1.73 \mathrm{~m}^{2}$ ). With this definition, 38 patients were classified as having rapidly and 114 patients as having slowly progressive disease. The urinary biomarker score again improved the predictive value of a model with age, sex, and eGFR included (AUC 0.79 [0.67-0.90] vs. 0.69 [0.57-0.81], respectively, $p=0.02$ ). Furthermore, the predictive value of the biomarker score was higher than that of the Mayo htTKV classification (AUC 0.75 [0.63-0.87] vs. 0.58 [0.47-0.70], $p=0.02$ ) and also compared to that of the PROPKD score, although this did again not reach formal statistical significance (AUC 0.75 [0.63-0.87] vs. 0.60 [0.48-0.73], $p=$ 0.09).

\section{Performance of Urinary Biomarker Score in an Independent Cohort}

We used another cohort of 104 patients with earlystage ADPKD [12] to test the predictive value of $\beta 2 \mathrm{MG}$ and MCP-1. Nine patients were excluded, who were also included in above analyses or had missing values, leaving 95 patients for analysis, of which $41.1 \%$ were female (age $39.6 \pm 11.1$ years, eGFR $78.6 \pm 29.6 \mathrm{~mL} / \mathrm{min} / 1.73 \mathrm{~m}^{2}$, and htTKV $852(510-1,212) \mathrm{mL} / \mathrm{m}$; online suppl. Table S6). During a follow-up time of $3.8 \pm 1.2$ years, annual change in eGFR was $-3.21 \pm 3.12 \mathrm{~mL} / \mathrm{min} / 1.73 \mathrm{~m}^{2}$ per year. We subsequently created tertiles of urinary excretion of $\beta 2 \mathrm{MG}$ and MCP $-1(<99,99-400,>400 \mu \mathrm{g} / 24 \mathrm{~h}$ and $<582$, $582-910,>910 \mathrm{ng} / 24 \mathrm{~h}$, respectively). Online supplemen- tary Figure S4 displays the proportion of patients of this independent cohort with rapidly or slowly progressive disease (annual change in eGFR of less than or equal to $-3.5 \mathrm{~mL} / \mathrm{min} / 1.73 \mathrm{~m}^{2}$ or greater than $-3.5 \mathrm{~mL} /$ $\mathrm{min} / 1.73 \mathrm{~m}^{2}$, respectively) and annual change in eGFR according to $\beta 2 \mathrm{MG}$ and MCP-1 excretion biomarker score. This score improved the predictive value of a model with age, sex, and eGFR included (AUC 0.66 [0.540.77 ] vs. 0.77 [0.68- 0.86$], p=0.02$ ). The urinary biomarker score performed equally compared to the Mayo $\mathrm{htTKV}$ classification in this population (AUC 0.72 [0.610.82 ] vs. 0.75 [0.64-0.85], $p=0.64)$. No data were available to calculate the PROPKD score, but information on $P K D$ mutation was available. The biomarker score tended to have a higher predictive value compared to $P K D$ mutation, although this did not reach statistical significance $(0.73[0.63-0.84]$ vs. $0.60[0.49-0.71], p=0.11)$.

\section{Discussion}

In the present study, we showed that the urinary excretion of in particular $\beta 2 \mathrm{MG}$ and MCP-1 predicts rapidly progressive disease in patients with ADPKD independent of TKV and PKD mutation. In our study population, the predictive value of a urinary biomarker score (based on tertiles of $\beta 2 \mathrm{MG}$ and MCP-1 excretion) was higher compared to that of the Mayo htTKV classification and equal to that of the PROPKD score.

We measured urinary tubular damage markers that represent inflammation and damage to different parts of the nephron. It is likely that in a chronic disease like AD$\mathrm{PKD}$, these processes occur simultaneously. Therefore, $>1$ urinary marker may have the ability to predict rapidly progressive disease and the predictive ability may also vary per patient. In this study, we chose to select those urinary markers that predict rapidly progressive disease in the overall study population when added to variables that are always available in routine clinical care (age, sex, and eGFR). When performing these analyses, urinary $\beta 2 \mathrm{MG}$ and MCP-1 showed the strongest associations with rapidly progressive disease, and these markers predicted rapidly progressive disease beyond age, sex, and eGFR, especially when added simultaneously.

In a previous study, we already showed that especially $\beta 2 \mathrm{MG}$ and MCP-1 had strong associations with annual change in eGFR [12], and the present study therefore corroborates these previous results. As $\beta 2 \mathrm{MG}$ reflects proximal tubular damage and MCP-1 reflects inflammation, these results suggest that the proximal tubule and inflam- 
mation are involved in the pathophysiology of ADPKD. Importantly, however, we caution against overinterpretation of our findings because one should be aware of the strengths and limitations of the various assays. The fact that associations between some markers and rapidly progressive disease are stronger should not lead to conclusions which parts of the renal tubule are more involved with cystogenesis. These results may in part be based on technical aspects, such as higher intra- and interassay coefficient of variation of the assay or less stability of the marker during frozen storage $[29,30]$.

Although TKV has repeatedly been shown to be a good predictor of the rate of eGFR decline, in our study it did not predict rapidly progressive disease when age, sex, eGFR, urinary $\beta 2 \mathrm{MG}$, and MCP- 1 were taken into account. Moreover, we demonstrated that a score based on urinary biomarkers alone had a higher predictive value for rapidly progressive disease than the Mayo htTKV classification. It could be that in our study, TKV was a less predictive biomarker because our study population was selected based on impaired eGFR, that is, it was enriched for subjects with rapidly progressive disease. Indeed, relatively few patients were classified as having Mayo htTKV class $1 \mathrm{~A} / \mathrm{B}$, and class $1 \mathrm{C} / \mathrm{D} / \mathrm{E}$ could not discriminate between patients with rapidly or slowly progressive disease (online suppl. Fig. S2). We repeated our analyses in an independent, unselected, observational cohort. In this cohort, we found that a score based on urinary biomarkers had a similar predictive value compared to the Mayo htTKV classification. Similarly, the PROPKD score (a score based on sex, the occurrence of hypertension and urological events before the age of 35 , and $P K D$ mutation) had an equal predictive value compared to the urinary biomarker score.

Our urinary biomarker score consists of 5 classes. Figure $2 \mathrm{~b}$ shows a graded association with rate of eGFR decline. These classes could be combined to a score with, for example, only 3 classes indicating low, intermediate, and high risk for rapidly progressive disease to make the score more clinically applicable. Similarly, for clinical purposes, the Mayo htTKV classification is divided in classes $1 \mathrm{~A} / \mathrm{B}, 1 \mathrm{C}$, and $1 \mathrm{D} / \mathrm{E}$ and the PROPKD score in a score of $0-3,4-6$, and 7-9, corresponding to low, intermediate, and high risk of reaching ESKD [11, 31].

These data indicate that although urinary biomarkers have an equal predictive value to htTKV class and genotype, optimal risk prediction may be achieved when information is taken into account on multiple variables, among which urinary biomarkers as well as htTKV and/ or genotype. In routine clinical care, however, informa-

Urinary Biomarkers in ADPKD tion on htTKV or PKD mutation is often not available because as yet they are relatively laborious and expensive to measure. Furthermore, with the PROPKD score, patients cannot be classified when they are younger than 35 years and not yet developed clinical events or when no mutation is detected. With a urinary biomarker score, that is relatively easy and inexpensive to obtain, all patients, also with earlier stage disease, can be classified. Furthermore, these urinary biomarkers are not ADPKD specific like htTKV or PKD mutation. This could be an advantage rather than a disadvantage because they may also reflect any damage on top of that caused by ADPKD, for instance due to environmental factors, which may lead to a more precise prediction of the rate of disease progression. Therefore, urinary biomarkers may be used as alternative to the more expensive and laborious volumetry and $P K D$ mutation analysis for risk prediction, but when other measures are available, they should also be taken into account.

Another advantage of urinary biomarkers is that they may rapidly respond to treatment, in contrast to TKV or $P K D$ mutation, and thereby serve as a surrogate of treatment response. We already showed in previous research that certain urinary biomarkers declined in response to 3 weeks of tolvaptan treatment [32]. Change in urinary excretion of tubular damage markers holds therefore promise to indicate treatment response in a certain patient. Future studies should investigate whether treatment-induced short-term changes in urinary tubular damage markers are associated with long-term outcome.

The present study has limitations. First, our cohort was selected for inclusion in a clinical trial with specific inclusion and exclusion criteria. We therefore did not include patients over the entire spectrum of the disease, but with later stage ADPKD [12], and consequently, there were not much differences between baseline characteristics of rapid and slow progressors. In this population with later stage ADPKD, eGFR indexed for age is already informative for risk prediction in contrast to patients with earlier stage ADPKD because eGFR can remain fairly stable, while cysts are already formed. Despite this, the urinary biomarkers were added to risk prediction in this population with later stage ADPKD. In a population where GFR indexed for age is less informative, the urinary biomarkers may then be of even more value. For these reasons, we also investigated the predictive value of a urinary biomarker score in an independent observational cohort that encompasses the entire spectrum of the disease, among which also earlier stage ADPKD, and found similar results. Second, for the 
measurement of urinary biomarkers, we used samples that were stored frozen, which may have influenced the results. However, previous research showed that $\beta 2 \mathrm{MG}$ and MCP-1 remain fairly stable during prolonged frozen storage $[33,34]$. It should be noted that the absolute values of the biomarker excretions hold for the present study as there may be differences between assays that are commercially available in other studies. In the future, the available assays need to be optimized and standardized to define specific cutoff values with high sensitivity and specificity before these biomarkers can be implemented in clinical care. Third, we defined rapid disease progression based on the mean kidney function decline in the study population, and results may be different when another definition is used. We therefore also defined rapid disease progression based on the quartile with the most rapid kidney function decline of the study population (annual change in eGFR of less than or equal to $-4.9 \mathrm{~mL} / \mathrm{min} / 1.73 \mathrm{~m}^{2}$ ) and found essentially similar results.

Strengths of this study are that we included ADPKD patients from which standardized follow-up data were available. Based on 13 fasting creatinine measurements over time, we were able to calculate reliable individual eGFR slopes using mixed models. Some assume that eGFR trajectories are not linear over time in patients with ADPKD, with eGFR that can remain fairly stable at young age [35]. In this study, however, we investigated patients with later stage disease. In such patients, eGFR slopes are reliable. In addition, we had data available to calculate the Mayo htTKV class and PROPKD score to allow comparisons, and we were able to validate our findings in an independent cohort. Last, all patients collected 24-h urine for biomarker assessment, which, due to the circadian rhythm in urinary excretion of these markers, is assumed to be better than spot urines that are used in most other studies [36].

In conclusion, urinary $\beta 2 \mathrm{MG}$ and $\mathrm{MCP}-1$ excretion have the ability to select patients with rapidly progressive ADPKD, with a predictive value comparable to or even higher than that of TKV and PKD mutation. Easy and inexpensive to measure urinary markers therefore hold promise to help identify patients with rapidly progressive disease, for instance, to be treated with disease-modifying agents.

\section{Acknowledgments}

The DIPAK Consortium is an inter-university collaboration in The Netherlands established to study ADPKD and to develop treatments for this disease.

For the present study, we acknowledge R.L. Kadijk for assistance at the outpatient clinic; P. Kappert, J. Grozema, and A. Sibeijn-Kuiper for assistance during MRI; B. Haandrikman and W. van Blitterswijk for assistance of laboratory procedures, and M.D.A. van Gastel, R. Bosman, R. Buiten, J. Heimovaara, M. Kaatee, M. de Jong, M. Levy, I. van Manen, C. Plate, L. Schepel, B. van der Slik, S.N. Voorrips, C.A. Wagenaar, and M.B. Wiertz for measuring TKVs.

\section{Disclosure Statement}

The authors have no conflicts of interest to declare.

\section{Funding Sources}

The DIPAK Consortium is sponsored by the Dutch Kidney Foundation (grants CP10.12 and CP15.01) and Dutch government (LSHM15018).

\section{Author Contributions}

R.T.G., E.M., and A.L.M.: research idea and study design; A.L.M., G.E.E., P.K., M.L., and D.J.M.P.: data acquisition; A.L.M., E.M., F.W.V., and R.T.G.: data analysis/interpretation; A.L.M. and R.T.G.: statistical analysis; R.T.G. and E.M.: supervision or mentorship; each author contributed important intellectual content during manuscript drafting or revision and accepts accountability for the overall work by ensuring that questions pertaining to the accuracy or integrity of any portion of the work are appropriately investigated and resolved.

\section{References}

1 Neumann HP, Jilg C, Bacher J, Nabulsi Z, Malinoc A, Hummel B, et al.; ElseKroener-Fresenius-ADPKD-Registry. Epidemiology of autosomal-dominant polycystic kidney disease: an in-depth clinical study for south-western Germany. Nephrol Dial Transplant. 2013 Jun;28(6): 1472-87.
2 Higashihara E, Nutahara K, Kojima M, Tamakoshi A, Yoshiyuki O, Sakai H, et al. Prevalence and renal prognosis of diagnosed autosomal dominant polycystic kidney disease in Japan. Nephron. 1998 Dec;80(4): $421-7$.

3 Grantham JJ, Torres VE, Chapman $\mathrm{AB}$, Guay-Woodford LM, Bae KT, King BF Jr, et al.; CRISP Investigators. Volume progression in polycystic kidney disease. N Engl J Med. 2006 May;354(20):2122-30.

4 Cornec-Le Gall E, Audrézet MP, Chen JM, Hourmant M, Morin MP, Perrichot R, et al. Type of PKD1 mutation influences renal outcomein ADPKD.JAm Soc Nephrol.2013 May; 24(6):1006-13. 
5 Torres VE, Chapman AB, Devuyst O, Gansevoort RT, Grantham JJ, Higashihara E, et al.; TEMPO 3:4 Trial Investigators. Tolvaptan in patients with autosomal dominant polycystic kidney disease. N Engl J Med. 2012 Dec; 367(25):2407-18.

6 Torres VE, Chapman AB, Devuyst O, Gansevoort RT, Perrone RD, Koch G, et al.; REPRISE Trial Investigators. Tolvaptan in LaterStage Autosomal Dominant Polycystic Kidney Disease. N Engl J Med. 2017 Nov;377(20): 1930-42.

7 Chapman AB, Devuyst O, Eckardt KU, Gansevoort RT, Harris T, Horie S, et al.; Conference Participants. Autosomal-dominant polycystic kidney disease (ADPKD): executive summary from a Kidney Disease: Improving Global Outcomes (KDIGO) Controversies Conference. Kidney Int. 2015 Jul; 88(1):17-27.

8 Gansevoort RT, Arici M, Benzing T, Birn H, Capasso G, Covic A, et al. Recommendations for the use of tolvaptan in autosomal dominant polycystic kidney disease: a position statement on behalf of the ERA-EDTA Working Groups on Inherited Kidney Disorders and European Renal Best Practice. Nephrol Dial Transplant. 2016 Mar;31(3):337-48.

9 Bhutani H, Smith V, Rahbari-Oskoui F, Mittal A, Grantham JJ, Torres VE, et al.; CRISP Investigators. A comparison of ultrasound and magnetic resonance imaging shows that kidney length predicts chronic kidney disease in autosomal dominant polycystic kidney disease. Kidney Int. 2015 Jul;88(1):146-51.

10 Irazabal MV, Rangel LJ, Bergstralh EJ, Osborn SL, Harmon AJ, Sundsbak JL, et al.; CRISP Investigators. Imaging classification of autosomal dominant polycystic kidney disease: a simple model for selecting patients for clinical trials. J Am Soc Nephrol. 2015 Jan; 26(1):160-72

11 Cornec-Le Gall E, Audrézet MP, Rousseau A Hourmant M, Renaudineau E, Charasse C, et al. The PROPKD Score: A New Algorithm to Predict Renal Survival in Autosomal Dominant Polycystic Kidney Disease. J Am Soc Nephrol. 2016 Mar;27(3):942-51.

12 Messchendorp AL, Meijer E, Boertien WE, Engels GE, Casteleijn NF, Spithoven EM, et al.; DIPAK Consortium. Urinary Biomarkers to Identify Autosomal Dominant Polycystic Kidney Disease Patients With a High Likelihood of Disease Progression. Kidney Int Rep. 2017 Oct;3(2):291-301

13 Meijer E, Drenth JP, d'Agnolo H, Casteleijn NF, de Fijter JW, Gevers TJ, et al.; DIPAK Consortium. Rationale and design of the DIPAK 1 study: a randomized controlled clinical trial assessing the efficacy of lanreotide to Halt disease progression in autosomal dominant polycystic kidney disease. Am I Kidney Dis. 2014 Mar;63(3):446-55.
14 Pei Y, Obaji J, Dupuis A, Paterson AD, Magistroni R, Dicks E, et al. Unified criteria for ultrasonographic diagnosis of ADPKD. J Am Soc Nephrol. 2009 Jan;20(1):205-12.

$15 \mathrm{Du}$ Bois D, Du Bois EF. A formula to estimate the approximate surface area if height and weight be known. 1916. Nutrition. 1989 SepOct:5(5):303-11; discussion 312-3.

16 Rossetti S, Hopp K, Sikkink RA, Sundsbak JL, Lee YK, Kubly V, et al. Identification of gene mutations in autosomal dominant polycystic kidney disease through targeted resequencing. J Am Soc Nephrol. 2012 May;23(5):91533.

17 Levey AS, Stevens LA, Schmid CH, Zhang YL, Castro AF 3rd, Feldman HI, et al.; CKD-EPI (Chronic Kidney Disease Epidemiology Collaboration). A new equation to estimate glomerular filtration rate. Ann Intern Med. 2009 May;150(9):604-12.

18 Gansevoort RT, Matsushita K, van der Velde M, Astor BC, Woodward M, Levey AS, et al.; Chronic Kidney Disease Prognosis Consortium. Lower estimated GFR and higher albuminuria are associated with adverse kidney outcomes. A collaborative metaanalysis of general and high-risk population cohorts. Kidney Int. 2011 Jul;80(1):93104.

19 Tofik R, Aziz R, Reda A, Rippe B, Bakoush O. The value of IgG-uria in predicting renal failure in idiopathic glomerular diseases. A longterm follow-up study. Scand J Clin Lab Invest. 2011 Apr;71(2):123-8.

20 Shin JR, Kim SM, Yoo JS, Park JY, Kim SK, Cho JH, et al. Urinary excretion of beta2-microglobulin as a prognostic marker in immunoglobulin A nephropathy. Korean J Intern Med. 2014 May;29(3):334-40.

21 Branten AJ, du Buf-Vereijken PW, Klasen IS, Bosch FH, Feith GW, Hollander DA, et al. Urinary excretion of beta2-microglobulin and $\mathrm{IgG}$ predict prognosis in idiopathic membranous nephropathy: a validation study. J Am Soc Nephrol. 2005 Jan;16(1):169-74.

22 Waanders F, van Timmeren MM, Stegeman CA, Bakker SJ, van Goor H. Kidney injury molecule-1 in renal disease. J Pathol. 2010 Jan; 220(1):7-16.

23 Hofstra JM, Deegens JK, Steenbergen EJ, Wetzels JF. Urinary excretion of fatty acidbinding proteins in idiopathic membranous nephropathy. Nephrol Dial Transplant. 2008 Oct;23(10):3160-5.

24 Kuusniemi AM, Lapatto R, Holmberg C, Karikoski R, Rapola J, Jalanko H. Kidneys with heavy proteinuria show fibrosis, inflammation, and oxidative stress, but no tubular phenotypic change. Kidney Int. 2005 Jul; 68(1):121-32.

25 Zhou LT, Lv LL, Pan MM, Cao YH, Liu H, Feng $\mathrm{Y}$, et al. Are Urinary Tubular Injury Markers Useful in Chronic Kidney Disease? A
Systematic Review and Meta Analysis. PLoS One. 2016;11(12):e0167334.

26 Tam FW, Riser BL, Meeran K, Rambow J, Pusey CD, Frankel AH. Urinary monocyte chemoattractant protein-1 (MCP-1) and connective tissue growth factor (CCN2) as prognostic markers for progression of diabetic nephropathy. Cytokine. 2009 Jul;47(1):37-42.

27 Torres VE, Abebe KZ, Chapman AB, Schrier RW, Braun WE, Steinman TI, et al.; HALTPKD Trial Investigators. Angiotensin blockade in late autosomal dominant polycystic kidney disease. N Engl J Med. 2014 Dec; 371(24):2267-76

28 Schrier RW, Abebe KZ, Perrone RD, Torres VE, Braun WE, Steinman TI, et al.; HALTPKD Trial Investigators. Blood pressure in early autosomal dominant polycystic kidney disease. N Engl J Med. 2014 Dec;371(24): 2255-66.

29 Nauta FL, Bakker SJ, Lambers Heerspink H, de Zeeuw D, van Oeveren W, Bilo H, et al. Effect of frozen storage on urinary concentration of kidney damage markers. Am J Kidney Dis. 2012 Apr;59(4):586-9.

30 Nauta FL, Scheven L, Meijer E, van Oeveren W, de Jong PE, Bakker SJ, et al. Glomerular and tubular damage markers in individuals with progressive albuminuria. Clin J Am Soc Nephrol. 2013 Jul;8(7):1106-14.

31 Gansevoort RT, Arici M, Benzing T, Birn H, Capasso G, Covic A, et al. Recommendations for the use of tolvaptan in autosomal dominant polycystic kidney disease: a position statement on behalf of the ERA-EDTA Working Groups on Inherited Kidney Disorders and European Renal Best Practice. Nephrol Dial Transplant. 2016 Mar;31(3):337-48.

32 Boertien WE, Meijer E, de Jong PE, ter Horst GJ, Renken RJ, van der Jagt EJ, et al. Shortterm Effects of Tolvaptan in Individuals With Autosomal Dominant Polycystic Kidney Disease at Various Levels of Kidney Function. Am J Kidney Dis. 2015 Jun;65(6): $833-41$.

33 Klasen IS, Reichert LJ, de Kat Angelino CM, Wetzels JF. Quantitative determination of low and high molecular weight proteins in human urine: influence of temperature and storage time. Clin Chem. 1999 Mar;45(3): 430-2.

34 Leemasawatdigul K, Gappa-Fahlenkamp H. Effect of storage conditions on the stability of recombinant human MCP-1/CCL2. Biologicals. 2011 Jan;39(1):29-32

35 Grantham JJ, Chapman AB, Torres VE. Volume progression in autosomal dominant polycystic kidney disease: the major factor determining clinical outcomes. Clin J Am Soc Nephrol. 2006 Jan;1(1):148-57.

36 McLean RM. Measuring population sodium intake: a review of methods. Nutrients. 2014 Oct;6(11):4651-62. 